\title{
Ozonioterapia como coadjuvante na irrigação do sistema de canais radiculares
}

\author{
Ozoniotherapy as a coadjuvant in the irrigation of the radicular channels system
}

La ozonioterapia como coadyuvante en el riego del sistema de canales radiculares

Recebido: 10/01/2021 | Revisado: 12/01/2021 | Aceito: 17/01/2021 | Publicado: 20/01/2021

\author{
Karina Gonzalez Camara Fernandes \\ ORCID: https://orcid.org/0000-0003-4644-3408 \\ Universidade Brasil, Brasil \\ E mail: karinagcf@yahoo.com.br \\ Dora Inés Kozusny-Andreani \\ ORCID: https://orcid.org/0000-0003.3579.6419 \\ Universidade Brasil, Brasil \\ E-mail: doraines@terra.com.br \\ Carla Roberta Tim \\ ORCID: https://orcid.org/0000-0002-4745-9375 \\ Universidade Brasil, Brasil \\ E-mail: carla.tim@universidadebrasil.edu.br \\ Lara Gimenez Bazan \\ ORCID: https://orcid.org/0000-0003-0535-5217 \\ Universidade Brasil, Brasil \\ E-mail: laragbazan98@gamil.com \\ Lucieni Cristina Trovati Moreti \\ ORCID: https://orcid.org/0000-0003-1368-6403 \\ Universidade Brasil, Brasil \\ E-mail: lucienimoreti@ hotmail.com \\ Luciana Estevam Simonato \\ ORCID: https://orcid.org/0000-0002-6413-5479 \\ Universidade Brasil, Brasil \\ E-mail: Luciana.simonato@universidadebrasiledu.br \\ Lívia Assis Garcia \\ ORCID: https://orcid.org/0000-0002-8343-3375 \\ Universidade Brasil, Brasi \\ E-mail: livia.assis@universidadebrasil.edu.br
}

\begin{abstract}
Resumo
Na prática clínica da odontologia, é evidenciado que apesar dos avanços na área da endodontia, agentes microbianos e erros técnicos são os principais fatores responsáveis pelo insucesso endodôntico. O preparo químico mecânico tem como objetivo remover tecido necrosado, vital, microrganismos e seus subprodutos do sistema de canais radiculares. No entanto, este preparo não é suficiente para a limpeza satisfatória em virtude da complexidade anatômica do sistema de canais radiculares, podendo gerar a permanência de microrganismos que poderão continuar viáveis e ativos, interferindo no processo de reparação dos tecidos perirradiculares. Desta forma se faz necessário a busca por soluções químicas irrigadoras ideais que possam proporcionar melhores resultados no tratamento endodôntico. Dentro deste contexto, o objetivo deste estudo é proporcionar, através de uma revisão narrativa, uma compreensão das evidências atuais disponíveis sobre a importância da utilização do ozônio $\mathrm{O}_{3}$, como coadjuvante à irrigação do sistema de canais radiculares na terapia endodôntica. A pesquisa realizada caracteriza-se como exploratório-descritiva, tratando-se da abordagem, consiste em uma pesquisa qualitativa. As buscas foram realizadas nas bases de dados bibliográficas do PubMed/MEDLINE, Biblioteca virtual da saúde (BVS), Web of Science e SciELO. As evidências encontradas nesse estudo direcionam que o ozônio é capaz de inativar microrganismos através da oxidação direta de seus componentes estruturais, podendo ser proposto como um coadjuvante em tratamentos endodônticos contra microrganismos (Gram positivo e Gram negativo) presentes na cavidade oral e canais radiculares. Entretanto, faz-se necessário ensaios clínicos controlados e randomizados para endossar sua eficácia e dosimetrias ideias na prevenção e tratamentos endodônticos.
\end{abstract}

Palavras-chave: Ozônio; Endodontia; Desinfecção.

\begin{abstract}
In the clinical practice of dentistry, it is evident that despite advances in the area of endodontics, microbial agents and technical errors are the main factors responsible for endodontic failure. The mechanical chemical preparation aims to remove necrotic, vital tissue, microorganismos and their by-products from the root canal system. However, this preparation is not sufficient for satisfactory cleaning due to the anatomical complexity of the root canal system, which
\end{abstract}


can generate the permanence of microorganisms that may remain viable and active, interfering in the repair process of periradicular tissues. Thus, it is necessary to search for ideal irrigating chemical solutions that can provide better results in endodontic treatment. Within this context, the objective of this study is to provide, through a narrative review, an understanding of the current available evidence on the importance of using ozone O3, as an adjunct to irrigation of the root canal system in endodontic therapy. The research carried out characterizes as exploratorydescriptive, in terms of the approach, it consists of a qualitative research. The searches were carried out in the bibliographic databases of PubMed / MEDLINE, Virtual Health Library (VHL), Web of Science and SciELO. In this study, they state that ozone is capable of inactivating microorganisms through the direct oxidation of its structural components, and can be proposed as an adjunct in endodontic treatments against microorganisms (Gram positive and Gram negative) present in the oral cavity and root canals. If necessary, controlled and randomized clinical trials to endorse its efficacy and dosimetry in prevention and endodontic treatments.

Keywords: Ozone; Endodontics; Disinfection.

\section{Resumen}

En la práctica clínica de la odontología, es evidente que a pesar de los avances en el área de la endodoncia, los agentes microbianos y los errores técnicos son los principales factores responsables del fracaso endodóntico. La preparación química mecánica tiene como objetivo eliminar el tejido vital, necrótico, los microrganismos y sus subproductos del sistema de conductos radiculares. Sin embargo, esta preparación no es suficiente para una limpieza satisfactoria debido a la complejidad anatómica del sistema de conductos radiculares, que puede generar la permanencia de microrganismos que pueden permanecer viables y activos, interfiriendo en el proceso de reparación de los tejidos perirradiculares. Por ello, es necesario buscar soluciones químicas de irrigación ideales que puedan proporcionar mejores resultados en el tratamiento de endodoncia. En este contexto, el objetivo de este estudio es proporcionar, a través de una revisión narrativa, la comprensión de la evidencia disponible actualmente sobre la importancia del uso del ozono O3, como complemento de la irrigación del sistema de conductos radiculares en la terapia endodóntica. La investigación realizada caracteriza como exploratorio-descriptivo, en cuanto al abordaje, consiste en una investigación cualitativa, las búsquedas se realizaron en las bases de datos bibliográficas de PubMed / MEDLINE, Virtual Health Library (BVS), Web of Science y SciELO. En este estudio afirman que el ozono es capaz de inactivar microrganismos a través de la oxidación directa de sus componentes estructurales, pudiendo ser propuesto como coadyuvante en tratamientos endodónticos contra microorganismos (Gram positivos y Gram negativos) presentes en la cavidad bucal y conductos radiculares. Si es necesario, ensayos clínicos controlados y aleatorizados para avalar su eficacia y dosimetría i en prevención y tratamientos de endodoncia.

Palabras clave: Ozono; Endodoncia; Desinfección.

\section{Introdução}

A cavidade oral possui uma das maiores taxas de microrganismos, ou seja, vírus, fungos, leveduras, protozoários e as bactérias, representando os maiores números (Siqueira Jr \& Roças, 2009). Uma vez que os dentes fazem parte da cavidade oral, a anatomia fechada da polpa dentária associado à dentina (esmalte e cemento) fornecem uma barreira primária eficaz contra a colonização microbiana (Gomes \& Herrera, 2018).

Em condições apropriadas, a microbiota oral normal pode dar origem a patógenos oportunistas se ocorrer acesso aos tecidos da polpa dentária, entretanto, alterações nessas barreiras físicas criam caminhos para as bactérias no sistema de canal radicular (Gomes \& Herrera, 2018). Rompidas as barreiras físicas e/ou biológicas, o estabelecimento da infecção dependerá da sobrevivência dos microrganismos dentro do espaço pulpar (Boch et al., 2016).

O tratamento endodôntico tem como finalidade prevenir ou curar uma doença infecciosa localizada, geralmente, no canal radicular, ápice ou periápice, garantindo a terapia do dente e sua função na cavidade oral. Entretanto, sabe-se que o preparo do canal radicular amplia o canal radicular principal promovendo a remoção mecânica da dentina infectada e simultaneamente favorece a penetração de irrigantes pelos canais, potencializando o processo de descontaminação (Estrela et al., 2014). No entanto, uma porcentagem significativa da superfície do canal radicular permanece intocada, independentemente dos instrumentos usados para o preparo mecânico (Siqueira Jr et al. 2018). Essas áreas não alcançadas podem abrigar os microrganismos dos protocolos de desinfecção do canal radicular (Gomes \& Herrera 2018; Silva et al., 2020).

Sabe-se que as bactérias Gram negativas são a principal causa de diversas doenças que acometem a polpa e a região perirradiculares (Gomes \& Herrera, 2018). Dentre os fatores de virulência das bactérias Gram-negativas, os lipopolissacarídeos (LPS/endotoxinas) são os principais desencadeadores da infecção endodôntica por causa de seus efeitos biológicos, que levam 
a uma interação complexa com fatores do hospedeiro, resultando em sintomatologia clínica, reação inflamatória e reabsorção de tecidos mineralizados (Martinho et al., 2010a; Martinho et al., 2010b; Gomes \& Herrera, 2018). Ainda, é conhecido que o ácido lipoteicóico (LTA), presente nas bactérias gram-positivas, também ocasionam lesões dessas regiões.

A literatura mostra que tanto o LPS como o LTA desencadeiam uma resposta inflamatória local, ativando monócitos/ macrófagos com consequente liberação de citocinas em locais perirradiculares que por sua vez estão diretamente relacionados à destruição do tecido (Gomes \& Herrera, 2018). As principais citocinas liberadas nesses processos inflamatórios são as IL-1 $\alpha$, IL-1 $\beta$, TNF- $\alpha$, IL-6, PGE2, IL-10 e MMPs e, consequentemente, estão associados à sintomatologia clínica (Busanello-Costa et al., 2020).

O hipoclorito de sódio $(\mathrm{NaOCl})$ é o irrigante de canal radicular mais comumente usado em endodontia devido a sua atividade antimicrobiana eficaz com consequente redução significativa nos níveis de endotoxinas (Zehnder, 2006; Fidalgo et al., 2010; Neelakantan et al., 2019). No entanto, vários estudos demonstraram que a eliminação bacteriana completa não pode ser alcançada de forma consistente com nenhum dos protocolos de desinfecção atuais (Siqueira Jr et al., 2018; Silva et al., 2020). Tem sido demonstrado que sua eficácia depende de sua concentração, temperatura, solução de pH e condições de armazenamento. Quanto maior a concentração do hipoclorito de sódio, mais grave é a reação potencial que pode ocorrer se o irrigante for levado inadvertidamente aos tecidos periapicais (Plotino et al., 2016).

Entretanto, a literatura mostra o hipoclorito de sódio, assim como a clorexidina, apresenta ação antimicrobiana e substantividade, ou seja, efeito residual, proporcionado ação prolongada (Noites et al., 2014). Além disso, o amplo uso de soluções irrigantes com cloro, como a hiploclorito de sódio, pode levar ao aparecimento de bactérias resistentes ao mesmo, que representam uma ameaça para a segurança microbiológica (Ding et al., 2019).

Dentro deste contexto, esforços têm sido realizados para desenvolver novas técnicas para fornecer desinfecção adicional para o sistema de canais radiculares Silva et al., (2020), como irrigação ultrassônica passiva (PUI) Dioguardi et al., (2018), terapia fotodinâmica (PDT) Eslami et al., (2019), Dantas et al., (2020), técnicas de irrigação contínua Dioguardi et al., (2018), Silva et al., (2020) e métodos de irrigação apical de pressão positiva e negativa (Dioguardi et al. 2018, Eslami et al., 2019, Silva et al., 2020). Ainda, estudos atuais têm investigados a atuação da ozonioterapia com o objetivo de aumentar a desinfecção no preparo químico mecânico de canais, assim, melhorar os resultados endodônticos (Nogales, et al., 2008; Huth et al., 2009; Farac et al., 2013; Hubbezoglu et al., 2014; Nogales et al., 2014; Sansriti et al., 2016).

A ozonioterapia é baseada na utilização do ozônio $\left(\mathrm{O}_{3}\right)$, um gás natural, que possui a capacidade de se dissociar rapidamente em água e liberar uma forma reativa de oxigênio capazes de oxidar estruturas celulares como lipídeos e proteínas insaturadas (Boch et al., 2016; Case et al., 2012). Essas espécies reativas, chamadas de ozonídeo, sinalizam e desencadeiam mudanças metabólicas que podem produzir efeitos antimicrobianos (Ding et al., 2019; Junior \& Lages., 2012). Alguns trabalhos descrevem que o ozônio atua em glicolipídeos, glicoproteínas ou certos aminoácidos, que estão presentes na membrana citoplasmática de microrganismos, tendo, portanto, eficácia antimicrobiana sem induzir resistência aos medicamentos (Rojas-Valencia, 2011, Boch et al., 2016; Case et al., 2012).

Assim, a ozonioterapia tem sido proposta como uma terapêutica que realiza a potencialização da fase de sanificação do sistema de canais radiculares, mostrando efeitos positivos na eliminação de alguns microorganismos (Nagayoshi et al., 2004; Noites et al., 2014; Hubbezoglu et al., 2014; Nogales et al., 2014).

Baseado no fato da do ozônio tem ganhado atenção na endodontia, sendo atualmente sugerido como uma técnica de desinfecção emergente e promissora, esse estudo teve por objetivo realizar uma revisão sobre o tema e fornecer a compreensão das evidências atuais sobre a ozonioterapia como uma provável terapia coadjuvante no preparo químico mecânico a ser utilizado na endodontia. 


\section{Metodologia}

Trata-se de uma revisão narrativa da literatura, de caráter descritivo exploratória, de natureza qualitativa, o processo de busca foi realizado de forma independente por dois revisores (LA. e S.S.) (Pereira et al., 2018; Gonçalves et al., 2021). As bases de dados bibliográficas consultadas foram PubMed/MEDLINE, Biblioteca Virtual de Saúde (BVS) e SciELO. A busca se deu por artigos baseados em evidências publicados até 10 de dezembro de 2020 e as principais palavras-chave utilizadas para a revisão foram: ozonioterapia, tratamento endodôntico e desinfecção. Nenhuma limitação de idioma foi aplicada e os dados publicados em outros idiomas que não o inglês foram traduzidos usando sites e softwares de tradução.

\section{Resultados e Discussão}

É possível identificar os efeitos positivos do ozônio na endodontia desde os experimentos realizados por Hyslop et al., (1988), Filippi (2001), os quais constataram uma diminuição significativa na citotoxicidade para células orais após a utilização do gás ozônio em comparação com irrigantes endodônticos, hipoclorito de sódio a 2,25\% e clorexidina a 2\%. A partir de então, o ozônio começou a ser discutido como uma possível alternativa ou agente antimicrobiano complementar durante o tratamento do canal radicular.

Os primeiros estudos desenvolvidos por Nagayoshi et al. (2004) mostram que a água é capaz de eliminar microorganismos bucais Gram positivos e Gram negativos e destacam a vantagem pois o manuseio da água ozonizada é uma abordagem fácil e segura quando comparado a forma gasosa, exerce potência oxidante e sugere a utilização da mesma em soluções de instrumentos odontológicos. No mesmo ano, os autores avaliaram a atuação da água ozonizada sobre microorganismos Streptococcus muatns e Enterococcus faecalis inoculados em dentes bovinos e observaram que eficácia da mesma em microorganismos intratubulares semelhante ao efeito de hipoclorito de sódio a 2,5\%. Ainda, foi verificada a citotoxicidade da água ozonizada em fibroblastos e interessantemente foi observado que a atividade metabólica das células foram maiores quando receberam a água ozonizada e houve um decréscimo no número de células viáveis que receberam ação do hipoclorito de sódio.

No ano seguinte, Hems et al., (2005), avaliaram o potencial do ozônio em Enterococcus faecalis em biofilmes e em placas planquitônica com intuito de verificar sua efetividade como irrigante endodôntico. Utilizou-se o microrganismo de um isolado clínico e o mesmo foi tratado com água ozonizada, gás ozônio e como controle, utilizou a solução de hipoclorito de sódio a 2,5\%. Realizou-se diluições seriadas e a contagem das unidades formadoras de colônias (UFC/ml). Cabe destacar que o ozônio foi borbulhado por 30, 60, 120 e 240 segundos. Como conclusão do experimento, verificou-se que não houve influência da água ozonizada sobre os biofilmes, entretanto, a água ozonizada (240 segundos) foi eficiente em eliminar Enterococcus faecalis em forma planquitônica.

A eficácia do ozônio (água destilada, vinagre de maçã) aplicado em um sistema de limpeza ultrassônica contra Staphylococcus aureus foi verificado por (Estrela et al., 2006). Foi possível identificar que as soluções ozonizadas se apresentaram eficazes na eliminação do microrganismo analisado. Ainda, Estrela et al., (2007) verificaram a eficácia antimicrobiana da água ozonizada, ozônio gasoso, hipoclorito de sódio 2,5\% e clorexidina $2 \%$. O experimento foi realizado em dentes humanos que foram inoculados com Enterococcus faecalis durante 60 dias. A conclusão do estudo demonstrou a água ozonizada, hipoclorito a 2,5\%, clorexidina $2 \%$ e aplicação de ozônio gasoso não foram suficientes para inativar o microrganismo.

No tratamento do canal radicular, foi possível verificar que alguns estudos investigaram o efeito bactericida do ozônio em comparação ao hipoclorito de sódio. Muller et al., (2007), investigaram e compararam os efeitos do ozônio gasoso da terapia fotodinâmica (PDT) sob biofilme maduro de seis espécies a eficácia antimicrobiana de comparados ao hipoclorito de 
sódio e clorexidina. Os autores concluíram que uma única aplicação do ozônio gasoso e PDT apresentaram efeito mínimo sobre a viabilidade dos microorganismos organizados em forma de biofilme, ou seja, não foram capazes de eliminar completamente as bactérias de biofilmes.

A eficácia da água ozonizada como agente irrigante na eliminação de Enterococcus faecalis e Candida albicans em canais radiculares foram testadas no estudo de Cardoso et al., (2008). Os dentes foram instrumentados seguidos de irrigação com água ozonizada ou solução salina, sendo que esses foram avaliados no primeiro e 7 dias. Os resultados mostraram que a água ozonizada reduziu o número de $\mathrm{UFC} / \mathrm{mL}$ do microorganismos no primeiro dia de avaliação, entretanto, 7 dias após, houve um aumento dos microorganismos. Dessa forma, concluiu-se que a água ozonizada, como agente irrigante, reduziu significantemente o número dos microorganismos avaliados.

O estudo de Huth et al., (2009) verificaram a eficácia antimicrobiana da água ozonizada e do gás contra os microorganismos Enterococcus faecalis, Candida albicans, Septostreptococcus micros e Pseudomonas aeruginosa foram cultivadas em cultura planctônica ou em mono-espécies de biofilmes em canais radicularespor três semanas. As culturas foram expostas ao ozônio, hipoclorito de sódio $2,25 \%$ e $5,25 \%$, digluconato de clorexidina $2 \%$, peróxido de hidrogênio $3 \%$ e solução salina tamponada com fosfato (grupo controle) por 1 minuto. O gás ozônio foi aplicado aos biofilmes em dois ambientes experimentais, semelhante a um canal de difícil acesso e outro de acesso fácil. Após coleta dos resultados, concluíram que a água ozonizada e o gás (em alta concentração) foram eficazes, assim como hipoclorito de sódio e clorexidina, enquanto que o peróxiodo de hidrogênio foi menos eficaz.

Case et al., (2012), este estudo examinou os efeitos de gás ozônio distribuído em solução salina em biofilmes de Enterococcus faecalis em canais radiculares de dentes extraídos (raízes únicas) com e sem o uso de agitação ultrassônica passiva. A presença e pureza dos biofilmes foi confirmada usando microscopia eletrônica de varredura e cultura. Biofilmes foram tratados com solução salina (controle negativo), hipoclorito de sódio a 1\% por 120 segundos (controle positivo), ozônio (140 ppm ozônio no ar a 2 L / min entregue em solução salina usando uma cânula por 120 segundos), solução salina com ativação passiva com ultrassônica $\left(70 \mathrm{kHz}\right.$ e $200 \mathrm{~mW} / \mathrm{cm}^{2}$ aplicada com inserto 15 mantido passivamente dentro do canal, por 120 segundos) e ozônio seguido imediatamente por agitação ultrassônica. Após o tratamento, as amostras foram retiradas do biofilme e diluído em série para contagem de placas. A análise revelou que o hipoclorito de sódio a $1 \%$ foi o agente desinfetante mais eficaz seguido pelo ozônio.

Kaya et al., (2014), estudaram a eficácia antimicrobiana do plasma de baixa temperatura sob pressão atmosférica (LTAPP), ozônio gasoso, hipoclorito de sódio a $25 \%$ (controle positivo) e solução salina (controle negativo) em Enterococcus faecalis nas paredes do canal radicular e túbulos dentinários. A eficácia antimicrobiana foi avaliada realizando a contagem de unidades formadoras de colônias antes e depois de cada protocolo de irrigação. A amostragem microbiana demonstrou uma eficácia superior de LTAPP em comparação com $\mathrm{NaOCl}$ no terço médio do canal radicular, enquanto ambos tiveram efeitos semelhantes na região da coroa e terço apical. Concluíram que hipoclorito de sódio e LTAPP foram melhores do que o ozônio na região da coroa e partes médias dos canais radiculares. Essas descobertas sugerem que o LTAPP não tem efeitos térmicos e químicos e pode ser de grande auxílio no tratamento endodôntico.

Hubbezoglu et al., (2014) observaram o efeito antibacteriano do ozônio aquoso com diferentes concentrações e técnicas de aplicação (manual e ultrassônica) contra Enterococcus faecalis em canais radiculares humanos, 80 dentes unirradiculares foram selecionados, preparados e esterilizados. Enterococcus faecalis foi inoculado nos canais radiculares e mantido a $37^{\circ} \mathrm{C}$ por $24 \mathrm{~h}$. Os dentes foram divididos em quatro grupos principais, cada um com 20 dentes: grupo $\mathrm{NaOCl}$ (controle positivo); de água ozonizada de 8, 12 e 16 ppm. Os procedimentos de desinfecção foram realizados por $180 \mathrm{~s}$ para garantir a padronização de todos os grupos de trabalho. Os autores concluíram que a atividade bactericida de alta concentração de água ozonizada $(16$ ppm) combinada com a técnica de aplicação ultrassônica apresentou eficácia semelhante à do $\mathrm{NaOCl}$ 
$5,25 \%$ em canais radiculares.

Já, Boch et al., (2016) avaliaram o efeito antimicrobiano do gás ozônio em comparação ao etilenodiaminotetraacético (EDTA) a 20\% e hipoclorito de sódio a 3\% em dentes humanos que foram contaminados com Enterococcus faecalis por 72 horas. No estudo realizaram também associações utilizando hipoclorito de sódio e ozônio e EDTA e ozônio. As contagens de UFC foram mais baixas no grupo NaOCl-ozônio e no grupo EDTA-ozônio. Dentro das limitações do presente estudo, pode-se concluir que o ozônio pode reduzir a quantidade Enterococcus faecalis, organizado em um biofilme, significativamente. O uso coadjuvante de gás ozônio para aumentar o efeito de uma solução irrigante como o $\mathrm{NaOCl}$ pode ser sugerido, especialmente em casos de bactérias resistentes, infecções persistentes ou quando o $\mathrm{NaOCl}$ não pode ser usado, além de quando o forame apical estiver totalmente aberto ou reabsorvido.

Ainda Noites et al., (2014) avaliaram se a irrigação com hipoclorito de sódio, clorexidina e gás ozônio, isoladamente ou em combinação, foram eficazes contra Enterococcus faecalis e Candida albicans. Utilizaram dentes humanos recentemente extraídos com raiz única e inocularam os microorganismos e as soluções irrigadoras testadas foram: hipoclorito de sódio a 1, 3 e $5 \%$, clorexidina $0,2 \%$ e $2 \%$ e gás ozônio aplicado por diferentes períodos de tempo, o mecanismo de ação foi avaliado por citometria de fluxo, os resultados demonstraram que o hipoclorito de sódio, clorexidina e ozônio isoladamente foram ineficazes em eliminar complemtamente os microorganismos. A associação de clorexidina a 2\% seguida de gás ozônio por $24 \mathrm{~s}$ promoveu a eliminação completa de Enterococcus faecalis e Candida albicans, sugerindo que essa combinação pode ser vantajosa no tratamento de canais readiculares contaminados.

Recentemente, Silva et al., (2020), relataram que as concentrações mais altas e períodos mais longos de aplicação de ozônio permitem melhores resultados de desinfecção.

De acordo com a revisão sistemática de Silva et al., 2020, o ozônio isolado e em altas concentrações Huth et al., (2009) ou por um tempo prolongado Noites et al., (2014) obtiveram eficácia antimicrobiana comparáveis à solução de NaOCl. Ainda, destaca-se seu potencial quando associado à irrigação ultrassônica passiva Hubbezoglu et al., (2014), NaOCl Boch et al., (2016) ou clorexidina (Noites et al., 2014).

Assim, ozônio tem sido proposto como um agente de ação antimicrobiana tanto na forma de diluído como em gás sendo esses efetivos quando prescrito em uma concentração e tempo adequado, além de aplicado corretamente intracanal após os procedimentos tradicionais de limpeza, desinfecção e modelagem. Além do efeito antimicrobiano, o uso da intervenção do ozônio em substituição ao $\mathrm{NaOCl}$ também tem sido sugerido em outras condições clínicas: ápice reabsorvido e/ou forame aberto, devido à sua menor citotoxicidade, e nos casos de bactérias resistentes e infecções persistentes quando o $\mathrm{NaOCl}$ foi usado anteriormente (Boch et al. 2016).

Adicionalmente, pode-se observar estudos que a maioria laboratoriais que avaliaram a ação antimicrobiana do ozônio contra Enterococcus faecalis, uma bactéria Gram-positiva. Ainda, existem estudo com fungos, bactérias Gram-positivas e outras bactérias Gram-negativas (Nagayoshi et al. 2004, Huth et al. 2009, Noites et al. 2014). De acordo com Silva et al., 2020, o ozônio tem diferentes efeitos antimicrobianos de acordo com os diferentes grupos de bactérias (Gram positivas e Gram negativas). Uma vez que a estrutura das bactérias Gram-negativas contém lipopolissacarídeos (LPS) e fosfolipídios na membrana, esse grupo parece mais suscetível ao ozônio, uma vez que sua interação ocorre diretamente nessas estruturas.

Adicionalmente, no presente estudo foi possível verificar uma grande variabilidade entre os protocolos de aplicação de ozônio na endodontia, como por exemplo, diferentes equipamentos geradores de ozônio, concentração de ozônio, estado físico do ozônio e tempo e técnica de aplicação e concentrações de $\mathrm{NaOCl}(1-5,25 \%)$. Embora os estudos de laboratório sugiram um potencial promissor do ozônio na odontologia, como solução irrigadora não realizaram estudos clínicos até o momento. Uma vez que os efeitos antimicrobianos estejam fortemente relacionados à dose de terapia e ao tempo de aplicação, sugere-se que mais experimentos com objetivo de verificar a dosagem ótima de ozônio, assim o tempo de aplicação mais 
indicada para o tratamento endodôntico. Ainda, ensaios clínicos randomizados duplo-cegos, bem desenhados e conduzidos com tamanho de amostra adequado são necessários para garantir a aplicabilidade segura e eficaz da técnica. Ainda, com esses estudos clínicos, espera-se verificar o potencial do ozônio frente à carga bacteriana global em biofilmes fixados nas paredes dentinárias.

\section{Considerações Finais}

Baseado nas evidências demostradas, é plausível concluir que o ozônio é capaz de inativar microrganismos através da oxidação direta de seus componentes estruturais, podendo ser proposto como um coadjuvante em tratamentos no tratamento contra microrganismos (Gram positivo e Gram negativo) presentes na cavidade oral e canais radiculares. Entretanto, faz-se necessário ensaios clínicos controlados e randomizados para endossar sua eficácia e dosimetrias ideias na prevenção e tratamentos endodônticos.

Considerando os resultados promissores da utilização da ozonioterapia na irrigação do sistema de canais radiculares, destacamos a importância de novos ensaios experimentais e clínicos (controlados e randomizados) para o entendimento dos mecanismos moleculares e celulares envolvidos, e principalmente da dosimetria ideal para uma aplicação segura e efetiva e segura em seres humanos.

\section{Referências}

Boch, T., Tennert, C., Vach, K., Al-ahmad, A., Hellwig, E., \& Polydorou, O. (2016). Effect of gaseous ozone on Enterococcus faecalis biofilm-an in vitro study. Clinical Oral Investigations, 20, 1733-1739. https://doi.org/10.1007/S00784-015-1667-1

Busanello-Costa, M., Rennó, A. C. M., Martignago, C. C. S., Tim, C. R., Muniz, T. M. Q. \& Marcelino, C. C. C. (2020). Benefícios do Fator de Crescimento Epidérmico (EGF) associado a terapia de fotobiomodulação a LED no reparo tecidual de feridas cutâneas. Research, Society and Development, 9(10), 1-28. DOI: http://dx.doi.org/10.33448/rsd-v9i10.9369

Case, P. D., Bird, P. S., Kahler, W. A., George, R., \& Walsh, L. J. (2012). Treatment of root canal biofilms of Enterococcus faecalis with ozone gas and passive ultrasound activation. Journal of Endodontics. Chicago, 38(4), 523-526. https://doi.org/10.1016/j.joen.2011.12.020

Dantas, E. P. V., Martignago, C. C. S., Tim, c. R., Barros Filho, R. J. S., Neves, T. M. A. \& Assis, L. (2020). Fotobiomodulação como coadjuvante no tratamento na lesão pulmonar aguda decorrente da sepse. Research, Society and Development, 9(10), 1-20. DOI: http://dx.doi.org/10.33448/rsd-v9i10.9024

Ding, W., Jin, W., Cao, S., Zhou, X., Wang, C., Jiang, Q., Huang, H., Tu, R. S. F., \& Wang, Q. (2019). Ozone disinfection of chlorine-resistant bacteria in drinking water. Water Research, 339-349. https://doi.org/10.1016/j.watres.2019.05.014

Dioguardi, M., Di Gioia, G. D., Illuzzi, G., Laneve, E., Cocco, A., \& Troiano G. (2018). Endodontic irrigants: Different methods to improve efficacy and related problems. European Journal of Dentistry, 12 / Issue 3. https://doi.org/10.4103/ejd.ejd_56_18

Eslami, L. M., Vatanpour, M., Aminzadeh, N., Mehrvarzfar, P., \& Taheri S. (2019). The comparison of intracanal medicaments, diode laser and photodynamic therapy on removing the biofilm of Enterococcus faecalis and Candida albicans in the root canal system (ex-vivo study). Photodiagnosis and Photodynamic Therapy, 157-161. https://doi.org/10.1016/j.pdpdt.2019.01.033

Estrela, C., Estrela, C. R. A., Decurcio, D. A., Hollanda, A. C. B., \& Silva, J. A. (2007). Antimicrobial efficacy of ozonated water, gaseous ozone, sodium hypochlorite and chlorhexidine in infected human root canals. International Endodontic Journal. Oxford,40(2), 85-93. https://doi.org/10.1111/j.13652591.2006.01185.x

Estrela, C., Holland, R., Estrela, C. R. A., Alencar, A. H. G., Sousa-Neto M.D., \& Pécora J. D. (2014). Characterization of successful root canal treatment. Brazilian Dental Journal, 25(1), 3-11. http://dx.doi.org/10.1590/0103-6440201302356

Farac, R. V., Pizzolitto, A. C., Tanomaru, J. M. G., Morgental, R. D., Lima, R. K. P., \& Bonetti-Filho, I. (2013). Ex-vivo effect of intracanal medications based on Ozone and calcium hydroxide in root canals contaminated with Enterococcus faecalis. Brazilian Dental Journal, 24(2), 103-106. http://dx.doi.org/10.1590/0103-6440201301992

Fidalgo, T. K. S., Barcelos, R., Portela, M. B., Soares, R. M. A., Gleiser, R., \& Silva-Filho, F. C. (2010). Inhibitory activity of root canal irrigants against Candida albicans, Enterococcus faecalis and Staphylococcus aureus. https://doi.org/10.1590/S1806-83242010000400006

Filippi, A. (2001). The effects of ozonized water on epithelial wound healing (in German). Deutsche Zahnärztliche Zeitschrift, 56, 104-8. https://www.researchgate.net/publication/237555230

Gonçalves, S. R., Tim, C. R., Martignago, C. C. S., Silva, M. C. P., Anaruma, C. A., \& Garcia L. A. (2021). Potencial da terapia por fotobiomodulação no tratamento da atrofia do músculo esquelético. Research, Society and Development, 10(1), 1-12. DOI: http://dx.doi.org/10.33448/rsd-v10i1.8527 
Gomes, B., \& Herrera, D. R. (2018). Etiologic role of root canal infection in apical periodontitis and its relationship with clinical symptomatology. Brazilian Oral Research, 32, e69. https://doi.org/10.1590/1807-3107bor-2018.vol32.0069

Hems, R. S., Gulabivala, K., -L. Ng, Y., Ready, D., \& Spratt, D. A. (2005). An in vitro evaluation of the ability of ozone to kill a strain of Enterococcus faecalis. International Endodontic Journal, 38, 22-29. https://doi.org/ 10.1111/j.1365-2591.2004.00891.x.

Hyslop, P. A., Hinshaw, D. B., \& Halsey, W. A. Jr, et al. (1988). Mechanisms of oxidant-mediated cell injury. The glycolytic and mitochondrial pathways of ADP phosphorylation are major intracellular targets inactivated by hydrogen peroxide. The Journal of Biological Chemistry, $263,1665-1675$.

Hubbezoglu, I. (2014). Antibacterial efficacy of aqueous ozone in root canals infected by enterococcus faecalis. Jundishapur J Microbiol, 7(7), e11411. https://doi.org/10.5812/jjm.11411

Kaya, B. U., Kececi, A. D., \& Guldas, H. E., et al. (2014) Efficacy of endodontic applications of ozone and low-temperature atmospheric pressure plasma on root canals infected with enterococcus faecalis. Letters in Applied Microbiology, 58, 8-15. https://doi.org/10.1111/lam.12148

Martinho, F. C., Chiesa, W. M. M., Leite, F. R. M., Cirelli, J. A., \& Gomes, B. P. F. A. (2010). Antigenic Activity of Bacterial Endodontic Contents from Primary Root Canal Infection with Periapical Lesions against Macrophage in the Release of Interleukin-1b and Tumor Necrosis Factor a. JOE, 36(9). https://doi.org/10.1016/j.joen.2010.06.012

Martinho, F. C., Chiesa, W. M. M., Leite, F. R. M., Cirelli, J. A., \& Gomes B. P. F. A. (2010). Antigenicity of Primary Endodontic Infection against Macrophages by the Levels of PGE2 Production. JOE, 37(5). https://doi.org/10.1016/j.joen.2010.12.005

Muller, P., Guggenheim, B., \& Schmidlin, P. R. (2007). Efficacy of gasiform ozone and photodynamic therapy on a multispecies oral biofilm in vitro. Eur $J$ Oral Sci, 115, 77-80. https://doi.org/10.1111/j.1600-0722.2007.00418.x

NagayoshI, M., Fukuizumi, T., Kitamura, C., Yano, J., Terashita, M., \& Nishihara, T. (2004). Efficacy of ozone on survival and permeability of oral microorganisms. Oral Microbiology and Immunology. Kitakyushu, 19(4), 240-246. https://doi.org/10.1111/j.1399-302X.2004.00146.x

Neelakantan. P., Herrera, D. R., Pecorari, V. G. A., \& Gomes B. P. F. A. (2019). Endotoxin levels after chemomechanical preparation of root canals with sodium hypochlorite or chlorhexidine: a systematic review of clinical trials and meta-analysis. International Endodontic Journal, 52, 19-27. https://doi.org/10.1111/iej.12963

Nogales, C. G., Ferrari, P. H., Kantorovich, E. O., \& Lage-Marques, J. L. (2008). Ozone therapy in medicine and dentistry. The Journal of Contemporary Dental Practice. São Paulo, 9(4), 75-84.

Nogales, C. G., Ferreira, M. B., Lage-Marques, J. L., \& Antoniazzi, J. H. (2014). Comparison of the antimicrobial activity of three different concentrations of aqueous ozone on Pseudomonas aeruginosa, Staphylococcus aureus, and Enterococcus faecalis - in vitro study. Revista Española de Ozonoterapia, 4(1), 9-15.

Noites, R., Pina-Vaz, C., Rocha, R., Carvalho, M. F., Gonçalves, A., \& Pina-Vaz, I. (2014). Synergistic Antimicrobial Action of Chlorhexidine and Ozone in Endodontic Treatment. Hindawi Publishing Corporation BioMed Research International. http://dx.doi.org/10.1155/2014/592423

Pereira A.S.; Shitsuka D. M. ; Pereira F. J. \& Shitsuka R. (2018). Metodologia da pesquisa científica. [e-book]. Santa Maria. Ed.UAB/NTE/UFSM.https://repositorio.ufsm.br/bitstream/handle/1/15824/Lic_Computacao_Metodologia-Pesquisa-Cientifica.pdf?sequence=1

Rojas-Valencia, M. N. (2011). Research on ozone application as disinfectant and action mechanisms on wastewater microorganisms. In: Mendez-Vilas A, (Ed.), Science Against Microbial Pathogens: Communicating Current Research and Technological Advances. (pp. 263-71). Badajoz: Formatex, https://www.researchgate.net/publication/265050308

Tiwari, S., Avinash, A., Katiyar, S., Aarthi Iyer, A., \& Jain, S. (2017). Dental applications of ozone therapy: A review of literature. The Saudi Journal for Dental Research, 8, 105-111. http://dx.doi.org/10.1016/j.sjdr.2016.06.005

Silva, E. J. N. L., Prado, M. C., Soares, D. N., Hecksher, F., Martins, J. N. R. \& Fidalgo, T. K. S. (2020). The effect of ozone therapy in root canal desinfection: a systematic review. International Endodontic Journal, 53, 317-332. https://doi.org/10.1111/iej.13229

Siqueira Junior, J. F., Rôças, I. N. (2009). Diversity of endodontic microbiota revisited. J Dent Res, 88(11), 969-81.

Siqueira Junior, J. F., Rôças, I. N., Marceliano-Alves, M. F., Pérez, A. R., \& Ricucci, D. (2018). Unprepared root canal surface areas: causes, clinical implications, and therapeutic strategies. Critical Review Endodontic Therapy, 32(suppl), e65. https://doi.org/10.1590/1807-3107bor-2018.vol32.0065

Plotino, G., Cortese, T., Grande, N. M., Leonardi, D. P., Giorgio, G. D., Testarelli, L., \& Gambarini, G. (2016). New Technologies to Improve Root Canal Disinfection. Brazilian Dental Journal, 27(1), 3-8. http://dx.doi.org/10.1590/0103-6440201600726

Zehnder, M. (2006). Root canal irrigants. J Endod, 32(5), 389-98. https://doi.org/10.1016/j.joen.2005.09.014 